American Journal of Agricultural and Biological Sciences 3 (3): 534-543, 2008

ISSN 1557-4989

(C) 2008 Science Publications

\title{
Pollen Flavonoid/Phenolic Acid Composition of Four Species of Cactaceae and its Taxonomic Significance
}

\author{
${ }^{1}$ Norma Almaraz-Abarca, ${ }^{2}$ María G. Campos, ${ }^{1}$ Elí A. Delgado-Alvarado, ${ }^{1}$ José A. Ávila-Reyes, \\ ${ }^{1}$ Jesús Herrera-Corral, ${ }^{1}$ Laura S. González-Valdez, ${ }^{1}$ Nestor Naranjo-Jiménez, ${ }^{2}$ Christian Frigerio, \\ ${ }^{2}$ Ana F. Tomatas, ${ }^{2}$ Ana J. Almeida, ${ }^{2}$ Amélia Vieira and ${ }^{1}$ José N. Uribe-Soto \\ ${ }^{1}$ Centro Interdisciplinario de Investigación para el Desarrollo Integral Regional, \\ Instituto Politécnico Nacional, Unidad Durango, \\ Sigma s/n 20 de Noviembre II, Durango, Dgo. México, 34220 \\ ${ }^{2}$ Laboratório de Farmacognosia, Faculdade de Farmácia, Universidade de Coimbra, \\ 3000-295 Coimbra, Portugal
}

\begin{abstract}
The pollen flavonoid/phenolic acid composition of 14 individual of Stenocactus multicostatus subsp. zacatecasensis (Britton et Rose) U. Guzmán et Vázquez-Benitez, comb. et stat. nov., 11 of Echinocereus enneacanthus Engelmann, 8 of Echinocereus pectinatus (Scheidweiler) Engelmann and 12 of Mammillaria heyderi sensu lato (all belonging to Cactaceae) was analyzed by HPLC/DAD with the aim of determining the role of pollen profiles of flavonoid/phenolic acid as valuable markers in those taxa. The flavonoid/phenolic acid composition of pollen was evaluated within and among the taxa analyzed. The results suggest that (a) the flavonoid/phenolic acid profiles of these species are among the most complex reported, (b) that quercetin, kaempferol and herbacetin glycoside derivatives are the major phenols found in the pollen of these species of cactus, (c) that some intrapopulation variability is present in all four species and (d) that these pollen flavonoid/phenolic acid profiles tend to be species-specific, so that they can represent important taxonomic markers in Cactaceae.
\end{abstract}

Key words: Stenocactus multicostatus, Echinocereus enneacanthus, Echinocereus pectinatus, Mammillaria heyderi, pollen flavonoids

\section{INTRODUCTION}

Cactaceae is a native family of American Continent; its natural distribution is from southwestern Canada to Chile. However, Mexico is where this family reaches its highest level of genera and species diversity $^{[1]}$. The family Cactaceae has between 1500 and 1800 species in approximately 100 genera $^{[2]}$. It is estimated that in Mexico around 707 species and 58 genera are found ${ }^{[3]}$, with around 14 endemic genera and 400 endemic species ${ }^{[4]}$.

The delimitation of taxa of this family and the understanding of their taxonomic relationships becomes a hard task because of many species and genera pertaining to Cactaceae, have been partially described or the description has been based solely on one individual, has not been typified or has been made without denoting the origin ${ }^{[5,6]}$. That is the case of Stenocactus multicostatus subsp. zacatecasensis ${ }^{[5,6]}$,
Echinocereus enneacanthus, Echinocereus pectinatus and Mammillaria heyderi sensu lato $^{[5]}$. The great morphological variability and the high hybridization capacity are two additional factors making more difficult the taxonomy of these groups.

Stenocactus (= Echinofossulocactus), Mammillaria and Echinocereus belong to subfamily Cactoideae ${ }^{[7]}$. Stenocactus and Mammillaria share, according to morphological features, a closer taxonomic relationship, since both belong to tribe Cacteae and to subtribe Cactinae, than between either of them and Echinocereus, which belongs to tribe Echinocerea ${ }^{[5]}$.

Chemical features, like flavonoid profiles, have been considered as significant taxonomic markers in the delimitation of species of different plant families ${ }^{[8-12]}$. Almost every species of plant synthesizes some kinds of flavonoids. Pollen is a very important site of synthesis and accumulation of flavonoids and phenolic acids ${ }^{[13,14]}$. Recently, reports of the taxonomic

Corresponding Author: Norma Almaraz-Abarca, Centro Interdisciplinario de Investigación para el Desarrollo Integral Regional, Instituto Politécnico Nacional, Unidad Durango, Sigma s/n 20 de Noviembre II, Durango, Dgo. México, 34220 Tel/Fax: (52 618) 8142091 
significance of pollen flavonoid/phenolic acid profiles have appeared ${ }^{[14,15]}$, this is relevant especially for chemotaxonomic studies of Cactaceae, in which many elements are fixed in some dangerous status, since the determination of those profiles represent a non plant destructive analysis.

The present study was conducted to investigate the intra- and interspecific variations in pollen flavonoid/phenolic acid compositions among one species of Mammillaria, one species of Stenocactus and two species of Echinocereus and establish their taxonomic significance in these taxa of Cactaceae.

\section{MATERIALS AND METHODS}

Sampling: Pollen of one species of Stenocactus, two of Echinocereus and one de Mammillaria were collected in different locations of Durango, Mexico (Table 1). Information about environmental conditions where each taxon develops was compiled. The flavonoid/phenolic acid composition was interpreted considering the previous identification of material based on morphological markers. A voucher of each population collected was deposited at the Herbarium CIIDIR.

Table 1: Collection sites for Stenocactus multicostatus subsp. zacatecasensis, Mammillaria heyderi sensu lato, Echinocereus enneacanthus, and Echinocereus pectinatus

\begin{tabular}{|c|c|c|c|c|c|c|c|}
\hline Sample & No. Ref. & Species & Latitude N & Longitude W & Altitude (m) & Location & Date \\
\hline$\overline{1}$ & 559 & $\begin{array}{l}\text { Stenocactus multicostatus } \\
\text { subsp. zacatecasensis }\end{array}$ & $24^{\circ} 07^{\prime} 16.5^{\prime \prime}$ & $104^{\circ} 45^{\prime} 00.1^{\prime \prime}$ & 1969 & $\begin{array}{l}\text { Chupaderos, } \\
\text { Durango, Mex. }\end{array}$ & March 2005 \\
\hline 2 & 560 & $\begin{array}{l}\text { Stenocactus multicostatus } \\
\text { subsp. zacatecasensis }\end{array}$ & $24^{\circ} 07^{\prime} 16.5^{\prime \prime}$ & $104^{\circ} 45^{\prime} 00.1^{\prime}$, & 1969 & $\begin{array}{l}\text { Chupaderos, } \\
\text { Durango, Mex. }\end{array}$ & March 2005 \\
\hline 3 & 561 & $\begin{array}{l}\text { Stenocactus multicostatus } \\
\text { subsp. zacatecasensis }\end{array}$ & $24^{\circ} 07^{\prime} 16.5^{\prime \prime}$ & $104^{\circ} 45^{\prime} 00.1^{\prime}$, & 1969 & $\begin{array}{l}\text { Chupaderos, } \\
\text { Durango, Mex. }\end{array}$ & March 2005 \\
\hline 4 & 562 & $\begin{array}{l}\text { Stenocactus multicostatus } \\
\text { subsp. zacatecasensis }\end{array}$ & $24^{\circ} 07^{\prime} 16.5^{\prime \prime}$ & $104^{\circ} 45^{\prime} 00.1^{\prime \prime}$ & 1969 & $\begin{array}{l}\text { Chupaderos, } \\
\text { Durango, Mex. }\end{array}$ & March 2005 \\
\hline 5 & 563 & $\begin{array}{l}\text { Stenocactus multicostatus } \\
\text { subsp. zacatecasensis }\end{array}$ & $24^{\circ} 07^{\prime} 16.5^{\prime \prime}$ & $104^{\circ} 45^{\prime} 00.1^{\prime \prime}$ & 1969 & $\begin{array}{l}\text { Chupaderos, } \\
\text { Durango, Mex. }\end{array}$ & March 2005 \\
\hline 6 & 564 & $\begin{array}{l}\text { Stenocactus multicostatus } \\
\text { subsp. zacatecasensis }\end{array}$ & $24^{\circ} 07^{\prime} 16.5^{\prime \prime}$ & $104^{\circ} 45^{\prime} 00.1^{\prime \prime}$ & 1969 & $\begin{array}{l}\text { Chupaderos, } \\
\text { Durango, Mex. }\end{array}$ & March 2005 \\
\hline 7 & 565 & $\begin{array}{l}\text { Stenocactus multicostatus } \\
\text { subsp. zacatecasensis }\end{array}$ & $24^{\circ} 07^{\prime} 16.5^{\prime \prime}$ & $104^{\circ} 45^{\prime} 00.1^{\prime}$, & 1969 & $\begin{array}{l}\text { Chupaderos, } \\
\text { Durango, Mex. }\end{array}$ & March 2005 \\
\hline 8 & 566 & $\begin{array}{l}\text { Stenocactus multicostatus } \\
\text { subsp. zacatecasensis }\end{array}$ & $24^{\circ} 07^{\prime} 16.5^{\prime \prime}$ & $104^{\circ} 45^{\prime} 00.1^{\prime}$, & 1969 & $\begin{array}{l}\text { Chupaderos, } \\
\text { Durango, Mex. }\end{array}$ & March 2005 \\
\hline 9 & 568 & $\begin{array}{l}\text { Stenocactus multicostatus } \\
\text { subsp. zacatecasensis }\end{array}$ & $24^{\circ} 07^{\prime} 16.5^{\prime \prime}$ & $104^{\circ} 45^{\prime} 00.1^{\prime}$, & 1969 & $\begin{array}{l}\text { Chupaderos, } \\
\text { Durango, Mex. }\end{array}$ & March 2005 \\
\hline 10 & 569 & $\begin{array}{l}\text { Stenocactus multicostatus } \\
\text { subsp. zacatecasensis }\end{array}$ & $24^{\circ} 07^{\prime} 16.5^{\prime \prime}$ & $104^{\circ} 45^{\prime} 00.1^{\prime \prime}$ & 1969 & $\begin{array}{l}\text { Chupaderos, } \\
\text { Durango, Mex. }\end{array}$ & March 2005 \\
\hline 11 & 571 & $\begin{array}{l}\text { Stenocactus multicostatus } \\
\text { subsp. zacatecasensis }\end{array}$ & $24^{\circ} 07^{\prime} 16.5^{\prime \prime}$ & $104^{\circ} 45^{\prime} 00.1^{\prime \prime}$ & 1969 & $\begin{array}{l}\text { Chupaderos, } \\
\text { Durango, Mex. }\end{array}$ & March 2005 \\
\hline 12 & 572 & $\begin{array}{l}\text { Stenocactus multicostatus } \\
\text { subsp. zacatecasensis }\end{array}$ & $24^{\circ} 07^{\prime} 16.5^{\prime \prime}$ & $104^{\circ} 45^{\prime} 00.1^{\prime}$, & 1969 & $\begin{array}{l}\text { Chupaderos, } \\
\text { Durango, Mex. }\end{array}$ & March 2005 \\
\hline 13 & 573 & $\begin{array}{l}\text { Stenocactus multicostatus } \\
\text { subsp. zacatecasensis }\end{array}$ & $24^{\circ} 07^{\prime} 16.5^{\prime \prime}$ & $104^{\circ} 45^{\prime} 00.1^{\prime}$, & 1969 & $\begin{array}{l}\text { Chupaderos, } \\
\text { Durango, Mex. }\end{array}$ & March 2005 \\
\hline 14 & 574 & $\begin{array}{l}\text { Stenocactus multicostatus } \\
\text { subsp. zacatecasensis }\end{array}$ & $24^{\circ} 07^{\prime} 16.5^{\prime \prime}$ & $104^{\circ} 45^{\prime} 00.1^{\prime \prime}$ & 1969 & $\begin{array}{l}\text { Chupaderos, } \\
\text { Durango, Mex. }\end{array}$ & March 2005 \\
\hline 15 & 567 & $\begin{array}{l}\text { Mammillaria heyderi } \\
\text { sensu lato }\end{array}$ & $24^{\circ} 07^{\prime} 16.5^{\prime \prime}$ & $104^{\circ} 45^{\prime} 00.1^{\prime \prime}$ & 1969 & $\begin{array}{l}\text { Chupaderos, } \\
\text { Durango, México }\end{array}$ & March 2005 \\
\hline 16 & 576 & $\begin{array}{l}\text { Mammillaria heyderi } \\
\text { sensu lato }\end{array}$ & $24^{\circ} 07^{\prime} 16.5^{\prime \prime}$ & $104^{\circ} 45^{\prime} 00.1^{\prime \prime}$ & 1969 & $\begin{array}{l}\text { Chupaderos, } \\
\text { Durango, México }\end{array}$ & March 2005 \\
\hline 17 & 577 & $\begin{array}{l}\text { Mammillaria heyderi } \\
\text { sensu lato }\end{array}$ & $24^{\circ} 07^{\prime} 16.5^{\prime \prime}$ & $104^{\circ} 45^{\prime} 00.1^{\prime \prime}$ & 1969 & $\begin{array}{l}\text { Chupaderos, } \\
\text { Durango, México }\end{array}$ & March 2005 \\
\hline 18 & 578 & $\begin{array}{l}\text { Mammillaria heyderi } \\
\text { sensu lato }\end{array}$ & $24^{\circ} 07^{\prime} 16.5^{\prime \prime}$ & $104^{\circ} 45^{\prime} 00.1^{\prime \prime}$ & 1969 & $\begin{array}{l}\text { Chupaderos, } \\
\text { Durango, México }\end{array}$ & March 2005 \\
\hline 19 & 579 & $\begin{array}{l}\text { Mammillaria heyderi } \\
\text { sensu lato }\end{array}$ & $24^{\circ} 07^{\prime} 16.5^{\prime \prime}$ & $104^{\circ} 45^{\prime} 00.1^{\prime \prime}$ & 1969 & $\begin{array}{l}\text { Chupaderos, } \\
\text { Durango, México }\end{array}$ & March 2005 \\
\hline 20 & 580 & $\begin{array}{l}\text { Mammillaria heyderi } \\
\text { sensu lato }\end{array}$ & $24^{\circ} 07^{\prime} 16.5^{\prime \prime}$ & $104^{\circ} 45^{\prime} 00.1^{\prime \prime}$ & 1969 & $\begin{array}{l}\text { Chupaderos, } \\
\text { Durango, México }\end{array}$ & March 2005 \\
\hline 21 & 581 & $\begin{array}{l}\text { Mammillaria heyderi } \\
\text { sensu lato }\end{array}$ & $24^{\circ} 07^{\prime} 16.5^{\prime \prime}$ & $104^{\circ} 45^{\prime} 00.1^{\prime \prime}$ & 1969 & $\begin{array}{l}\text { Chupaderos, } \\
\text { Durango, México }\end{array}$ & March 2005 \\
\hline 22 & 582 & $\begin{array}{l}\text { Mammillaria heyderi } \\
\text { sensu lato }\end{array}$ & $24^{\circ} 07^{\prime} 16.5^{\prime \prime}$ & $104^{\circ} 45^{\prime} 00.1^{\prime \prime}$ & 1969 & $\begin{array}{l}\text { Chupaderos, } \\
\text { Durango, México }\end{array}$ & March 2005 \\
\hline 23 & 583 & $\begin{array}{l}\text { Mammillaria heyderi } \\
\text { sensu lato }\end{array}$ & $24^{\circ} 07^{\prime} 16.5^{\prime \prime}$ & $104^{\circ} 45^{\prime} 00.1^{\prime \prime}$ & 1969 & $\begin{array}{l}\text { Chupaderos, } \\
\text { Durango, México }\end{array}$ & March 2005 \\
\hline 24 & 584 & Mammillaria heyderi & $24^{\circ} 07^{\prime} 16.5^{\prime \prime}$ & $104^{\circ} 45^{\prime} 00.1^{\prime \prime}$ & 1969 & Chupaderos, & March 2005 \\
\hline
\end{tabular}


Am. J. Agri. \& Biol. Sci., 3 (3): 534-543, 2008

Table 1: continue

\begin{tabular}{|c|c|c|c|c|c|c|c|}
\hline 25 & 585 & $\begin{array}{l}\text { sensu lato } \\
\text { Mammillaria heyderi } \\
\text { sensu lato }\end{array}$ & $24^{\circ} 07^{\prime} 16.5^{\prime}$ & $104^{\circ} 45^{\prime} 00.1^{\prime \prime}$ & 1969 & $\begin{array}{l}\text { Durango, México } \\
\text { Chupaderos, } \\
\text { Durango, México }\end{array}$ & March 2005 \\
\hline 26 & 586 & $\begin{array}{l}\text { Mammillaria heyderi } \\
\text { sensu lato }\end{array}$ & $24^{\circ} 07^{\prime} 16.5^{\prime}$ & $104^{\circ} 45^{\prime} 00.1^{\prime \prime}$ & 1969 & $\begin{array}{l}\text { Chupaderos, } \\
\text { Durango, México }\end{array}$ & March 2005 \\
\hline 27 & 609 & Echinocereus enneacanthus & $25^{\circ} 01^{\prime} 19.6^{\prime \prime}$ & $103^{\circ} 35^{\prime} 44.5^{\prime \prime}$ & 1550 & $\begin{array}{l}\text { Vallecillos, } \\
\text { Cuencamé, Durango }\end{array}$ & March 2005 \\
\hline 28 & 610 & Echinocereus enneacanthus & $25^{\circ} 01 ' 19.6 \prime$ & $103^{\circ} 35^{\prime} 44.5^{\prime \prime}$ & 1550 & $\begin{array}{l}\text { Vallecillos, } \\
\text { Cuencamé, Durango }\end{array}$ & March 2005 \\
\hline 29 & 611 & Echinocereus enneacanthus & $25^{\circ} 01^{\prime} 19.6^{\prime}$, & $103^{\circ} 35^{\prime} 44.5^{\prime}$, & 1550 & $\begin{array}{l}\text { Vallecillos, } \\
\text { Cuencamé, Durango }\end{array}$ & March 2005 \\
\hline 30 & 612 & Echinocereus enneacanthus & $25^{\circ} 01^{\prime} 19.6^{\prime \prime}$ & $103^{\circ} 35^{\prime} 44.5^{\prime \prime}$ & 1550 & Cañón de San Diego, & March 2005 \\
\hline 31 & 638 & Echinocereus enneacanthus & $26^{\circ} 41^{\prime} 17.7^{\prime \prime}$ & $103^{\circ} 44^{\prime} 50.5^{\prime \prime}$ & 1160 & Mapimí, Durango & April 2005 \\
\hline 32 & 639 & Echinocereus enneacanthus & $26^{\circ} 41^{\prime} 17.7^{\prime \prime}$ & $103^{\circ} 44^{\prime} 50.5^{\prime \prime}$ & 1160 & Mapimí, Durango & April 2005 \\
\hline 33 & 641 & Echinocereus enneacanthus & $26^{\circ} 41^{\prime} 17.7^{\prime \prime}$ & $103^{\circ} 44^{\prime} 50.5^{\prime \prime}$ & 1160 & Mapimí, Durango & April 2005 \\
\hline 34 & 643 & Echinocereus enneacanthus & $26^{\circ} 42^{\prime} 06.1^{\prime \prime}$ & $103^{\circ} 38^{\prime} 04.5^{\prime \prime}$ & 1150 & Mapimí, Durango & April 2005 \\
\hline 35 & 644 & Echinocereus enneacanthus & $26^{\circ} 41^{\prime} 17.7^{\prime \prime}$ & $103^{\circ} 44^{\prime} 50.5^{\prime \prime}$ & 1160 & Mapimí, Durango & April 2005 \\
\hline 36 & 645 & Echinocereus enneacanthus & $26^{\circ} 41^{\prime} 17.7^{\prime \prime}$ & $103^{\circ} 44^{\prime} 50.5^{\prime \prime}$ & 1160 & Mapimí, Durango & April 2005 \\
\hline 37 & 646 & Echinocereus enneacanthus & $26^{\circ} 41^{\prime} 17.7^{\prime \prime}$ & $103^{\circ} 44^{\prime} 50.5^{\prime \prime}$ & 1160 & Mapimí, Durango & April 2005 \\
\hline 38 & 630 & Echinocereus pectinatus & $24^{\circ} 07^{\prime} 16.5^{\prime \prime}$ & $104^{\circ} 45^{\prime} 00.1^{\prime \prime}$ & 1969 & Chupaderos, Durango & April 2005 \\
\hline 39 & 631 & Echinocereus pectinatus & $24^{\circ} 07^{\prime} 16.5^{\prime \prime}$ & $104^{\circ} 45^{\prime} 00.1^{\prime \prime}$ & 1969 & Chupaderos, Durango & April 2005 \\
\hline 40 & 632 & Echinocereus pectinatus & $24^{\circ} 07^{\prime} 16.5^{\prime \prime}$ & $104^{\circ} 45^{\prime} 00.1^{\prime \prime}$ & 1969 & Chupaderos, Durango & April 2005 \\
\hline 41 & 634 & Echinocereus pectinatus & $24^{\circ} 05^{\prime} 00^{\prime \prime}$ & $104^{\circ} 40^{\prime} 30^{\prime \prime}$ & 1940 & $5 \mathrm{Km}$ N Durango-Parral & April 2005 \\
\hline 42 & 635 & Echinocereus pectinatus & $24^{\circ} 05^{\prime} 00^{\prime \prime}$ & $104^{\circ} 40^{\prime} 30^{\prime \prime}$ & 1940 & $5 \mathrm{Km}$ N Durango-Parral & April 2005 \\
\hline 43 & 636 & Echinocereus pectinatus & $24^{\circ} 05^{\prime} 00^{\prime \prime}$ & $104^{\circ} 40^{\prime} 30^{\prime \prime}$ & 1949 & $5 \mathrm{Km} \mathrm{N}$ Durango-Parral & April 2005 \\
\hline 44 & 637 & Echinocereus pectinatus & $24^{\circ} 05^{\prime} 00^{\prime \prime}$ & $104^{\circ} 40^{\prime} 30^{\prime \prime}$ & 1940 & $5 \mathrm{Km} N$ Durango-Parral & April 2005 \\
\hline 45 & 658 & Echinocereus pectinatus & $25^{\circ} 01^{\prime} 19.6^{\prime \prime}$ & $103^{\circ} 35^{\prime} 44.5^{\prime \prime}$ & 1550 & Cuencamé, Durango & April 2005 \\
\hline
\end{tabular}

HPLC/DAD analysis: Ten milligrams of dried pollen of each sample were sonicated in an ethanol-water solution $(1 \mathrm{~mL}, 50 \% \mathrm{v} / \mathrm{v})$ for $60 \mathrm{~min}$. The resultant mixtures were centrifuged at $15269 \mathrm{~g}$ for $10 \mathrm{~min}$ and the supernatants were used for HPLC/DAD analysis as previously described ${ }^{[14]}$. Extracts $(20 \mu \mathrm{L})$ were analyzed on a Gilson 170 and Waters Spherisorb ODS2 $(5 \mu \mathrm{m})$ column $(4.6 \times 250 \mathrm{~mm})$ by an acidified acetonitrile-water gradient. Standard chromatograms were plotted at $\lambda_{\max } 260$ and $340 \mathrm{~nm}$. Spectral data for all peaks were accumulated in the range $220-400 \mathrm{~nm}$ using DAD (Gilson 170). The phenolic profile of each sample was made up of all compounds resolved in its respective chromatogram. Each compound was treated as a single chemical character. The structural information was made by direct comparison of retention time and absortion spectra with standards and according to the information compiled by Campos and Markham $^{[16]}$.

Data analysis: The diversity of the profile of Flavonoid/phenolic acid for each taxon was derived using the Shannon index $(\mathrm{H})$.

A presence-absence matrix formed by all individual samples vs. all resolved compounds (45 individuals vs. 46 compounds) was analyzed using the UPGMA method (clustering program NTSYSpc $1.8^{[17]}$.

\section{RESULTS AND DISCUSSION}

HPLC/DAD analysis: A total of 46 compounds were resolved by HPLC/DAD (Table 2). Flavonoids and phenolic acids were the only two classes of phenolics found in the pollen of all species of Cactaceae analyzed; 3-O-flavonolglycosides were the major structures among the flavonoids. The analysis revealed 16 kaempferol derivatives, 7 quercetin derivatives, 5 herbacetin derivatives, 4 unidentified flavonoids, 3 unidentified phenols and 11 phenolic acid derivatives.

Flavonoids are present in pollen of many species of Angiosperm and Gymnosperm and in spores of moss and ferns ${ }^{[18]}$. Particularly kaempferol and quercetin are two required flavonols to pollen tube germination and growth in tobacco ${ }^{[18]}$, petunia and maize ${ }^{[19]}$. However, these two flavonols have not been shown to carry a similar function in Arabidopsis ${ }^{[20]}$. The abundance of kaempferol and quercetin derivatives in pollen of Stenocactus multicostatus subsp. zacatecasensis, Echinocereus pectinatus, E. enneacanthus and Mammillaria heyderi sensu lato could suggest that those flavonols could serve an essential function during reproduction of these species of Cactaceae.

Phenolic acids derivatives were more abundant in the pollen of Stenocactus multicostatus subsp. zacatecasensis (8 structures, Table 3 ) than in the pollen of Mammillaria heyderi sensu lato (2 structure, 
Am. J. Agri. \& Biol. Sci., 3 (3): 534-543, 2008

Table 2: Major phenols found in pollen of Stenocactus multicostatus subsp. zacatecasensis, Echinocereus pectinatus, Echinocereus enneacanthus and Mammillaria heyderi sensu lato

\begin{tabular}{|c|c|c|}
\hline No.of compound & Retention time $(\mathrm{min})(\mathrm{X} \pm \mathrm{SD})$ & Chemical identification \\
\hline $\bar{F} 1$ & $25.73 \pm 0.010$ & Unidentified phenol \\
\hline $\mathrm{F} 2$ & $29.93 \pm 0.030$ & Unidentified phenol \\
\hline F3 & $30.65 \pm 0.020$ & Unidentified phenol \\
\hline $\mathrm{F} 4$ & $32.24 \pm 0.020$ & Kaempferol-3-O-glycoside \\
\hline F5 & $32.54 \pm 0.020$ & Kaempferol-3-O-glycoside \\
\hline F6 & $32.56 \pm 0.033$ & Kaempferol-3-O-glycoside-7-substituted \\
\hline F7 & $32.68 \pm 0.050$ & Phenolic acid \\
\hline F8 & $33.91 \pm 0.000$ & Kaempfero-3-O-glycoside \\
\hline F9 & $34.16 \pm 0.020$ & Herbacetin-3-O-glycoside \\
\hline F10 & $34.18 \pm 0.010$ & Phenolic acid \\
\hline F11 & $35.73 \pm 0.090$ & Phenolic acid \\
\hline F12 & $35.82 \pm 0.030$ & Herbacetin-3-O-glycoside \\
\hline F13 & $36.35 \pm 0.030$ & Herbacetin-3-O-glycoside \\
\hline F14 & $36.40 \pm 0.000$ & Phenolic acid \\
\hline F15 & $36.56 \pm 0.040$ & Kaempferol-3-O-glycoside \\
\hline F16 & $36.80 \pm 0.000$ & Unidentified flavonol \\
\hline F17 & $37.28 \pm 0.020$ & Quercetin-3-O-glycoside \\
\hline F18 & $37.54 \pm 0.020$ & Herbcetin-3-O-glycoside \\
\hline F19 & $37.76 \pm 0.000$ & Herbacetin-3-O-glycoside \\
\hline F20 & $37.77 \pm 0.030$ & Quercetin-3-O-glycoside \\
\hline F21 & $37.80 \pm 0.010$ & Kaempferol-3-O-glycoside \\
\hline F22 & $37.82 \pm 0.010$ & Unidentified flavonoid \\
\hline F23 & $37.88 \pm 0.040$ & Quercetin-3-O-glycoside \\
\hline F24 & $38.20 \pm 0.040$ & Kaempferol-3-O-glycoside (probably susbtituted in ring $\mathrm{A}$ ) \\
\hline $\mathrm{F} 25$ & $38.24 \pm 0.020$ & Kaempferol-3-O-glycoside \\
\hline F26 & $38.61 \pm 0.040$ & Kaempferol-3-O-glycoside \\
\hline F27 & $38.89 \pm 0.040$ & Quercetin-3-O-glycoside \\
\hline F28 & $38.96 \pm 0.020$ & Inidentified flavonol \\
\hline F29 & $39.08 \pm 0.030$ & Pheolic acid \\
\hline F30 & $39.12 \pm 0.050$ & Kaempferol-3-O-glycoside \\
\hline F31 & $39.30 \pm 0.020$ & Kaempferol-3-O-glycoside \\
\hline F32 & $39.42 \pm 0.000$ & Unidentified flavonol \\
\hline F33 & $39.88 \pm 0.026$ & Kaempferol-3-O-glycoside \\
\hline F34 & $40.10 \pm 0.032$ & Kaempferol-3-O-glycoside \\
\hline F35 & $40.10 \pm 0.040$ & Quercetin-3-O-glycoside \\
\hline F36 & $40.56 \pm 0.020$ & Quercetin-3-O-glycoside \\
\hline F37 & $41.17 \pm 0.050$ & Kaempferol-3-O-glycoside \\
\hline F38 & $41.55 \pm 0.020$ & Quercetin-3-O-glycoside \\
\hline F39 & $42.56 \pm 0.020$ & Kaempferol-3-O-glycoside-7-sustituted \\
\hline F40 & $42.57 \pm 0.050$ & Kaempferol-3-O-glycoside \\
\hline F41 & $42.63 \pm 0.000$ & Phenolic acid \\
\hline $\mathrm{F} 42$ & $42.90 \pm 0.010$ & Phenolic acid \\
\hline $\mathrm{F} 43$ & $43.19 \pm 0.010$ & Phenolic acid \\
\hline F44 & $43.92 \pm 0.020$ & Phenolic acid \\
\hline F45 & $44.17 \pm 0.000$ & Phenolic acid \\
\hline F46 & $53.30 \pm 0.020$ & Phenolic acid \\
\hline
\end{tabular}

Table 4) and Echinocereus pectinatus (one structure, Table 5), while in the pollen of Echinocereus enneacanthus no phenolic acid was found (Table 6).

The pollen phenolic profiles of four all species of Cactaceae analyzed were relatively complex (22 phenolic compounds in Stenocactus multicostatus subsp. zacatecasensis, 17 in Mammillaria heyderi sensu lato, 17 in E. pectinatus and 9 in Echinocereus enneacanthus). The phenols richness found in this study represents a contrast with other reports on pollen phenols obtained by the same method; for example those of Zea mays with six compounds, Bidens odorata with three ${ }^{[15]}$; Eucalyptus globulus with seven and Erica australis with two ${ }^{[14]}$. A similar complex composition has been found in the perianth parts of Echinocereus triglochidiatus var. gurneyi (Cactaceae), but in contrast to pollen composition, dihydroflavonols and dihydroflavonol 7-O-gycosides, besides flavonol glycosides were present ${ }^{[21]}$. Other 
Am. J. Agri. \& Biol. Sci., 3 (3): 534-543, 2008

Table 3: Major phenols found in the pollen of Stenocactus multicostatus subsp. zacatecasensis

\begin{tabular}{|c|c|c|c|c|c|c|c|c|c|c|c|c|c|c|c|c|}
\hline \multirow{2}{*}{$\begin{array}{l}\text { No. of } \\
\text { compound }\end{array}$} & \multirow[b]{2}{*}{ Compound } & \multirow{2}{*}{$\begin{array}{l}\text { Retention } \\
\text { time (min) } \\
(\mathrm{X} \pm \mathrm{SD})\end{array}$} & \multicolumn{14}{|c|}{$\begin{array}{l}\text { Samples } \\
\text { Stenocactus multicostatus subsp. zacatecasensis }\end{array}$} \\
\hline & & & 559 & 560 & 561 & 562 & 563 & 564 & 565 & 566 & 568 & 569 & 571 & 572 & 573 & 574 \\
\hline F5 & Kaempferol-3-O-glycoside & $32.54 \pm 0.020$ & 1 & 1 & 1 & 1 & 1 & 1 & 1 & 1 & 1 & 1 & 1 & 1 & 1 & 1 \\
\hline F13 & Herbacetin-3-O-glycoside & $36.35 \pm 0.030$ & 1 & 1 & 1 & 1 & 1 & 1 & 1 & 1 & 1 & 1 & 1 & 1 & 1 & 1 \\
\hline F14 & Phenolic acid & $36.40 \pm 0.00$ & 1 & 1 & 1 & 1 & 1 & 1 & 1 & 1 & 1 & 1 & 1 & 1 & 1 & 1 \\
\hline F17 & Quercetin-3-O-glycoside & $37.28 \pm 0.02$ & 1 & 1 & 1 & 1 & 1 & 1 & 1 & 1 & 1 & 1 & 1 & 1 & 1 & 1 \\
\hline F18 & Herbacetin-3-O-glycoside & $37.54 \pm 0.02$ & 1 & 1 & 1 & 1 & 1 & 1 & 1 & 1 & 1 & 1 & 1 & 1 & 1 & 1 \\
\hline F23 & Quercetin-3-O-glycoside & $37.88 \pm 0.04$ & 1 & 1 & 1 & 1 & 1 & 1 & 1 & 1 & 1 & 1 & 1 & 1 & 1 & 1 \\
\hline F24 & $\begin{array}{l}\text { Kaempferol-3- } O \text {-glycoside } \\
\text { (Probably substituted in ring A) }\end{array}$ & $38.20 \pm 0.04$ & 0 & 0 & 0 & 1 & 1 & 0 & 1 & 0 & 1 & 1 & 1 & 1 & 0 & 0 \\
\hline F26 & Kaempferol-3-O-glycoside & $38.61 \pm 0.04$ & 1 & 1 & 1 & 1 & 1 & 1 & 1 & 1 & 1 & 1 & 1 & 1 & 1 & 1 \\
\hline F27 & Quercetin-3-O-glycoside & $38.96 \pm 0.02$ & 1 & 1 & 1 & 1 & 1 & 1 & 1 & 1 & 1 & 1 & 1 & 1 & 1 & 1 \\
\hline F29 & Phenolic acid & $39.08 \pm 0.03$ & 1 & 0 & 0 & 0 & 0 & 0 & 0 & 0 & 0 & 0 & 0 & 0 & 0 & 0 \\
\hline F31 & Kaempferol-3-O-glycoside & $39.30 \pm 0.02$ & 1 & 1 & 1 & 1 & 1 & 1 & 1 & 1 & 1 & 1 & 1 & 1 & 1 & 1 \\
\hline F32 & Unidentified flavonoid & $39.42 \pm 0.00$ & 1 & 0 & 0 & 0 & 0 & 0 & 0 & 0 & 0 & 0 & 0 & 0 & 0 & 0 \\
\hline F34 & Kaempferol-3-O-glycoside & $40.10 \pm 0.032$ & 1 & 1 & 1 & 1 & 1 & 1 & 1 & 1 & 1 & 1 & 1 & 1 & 1 & 1 \\
\hline F37 & Kaempferol-3-O-glycoside & $41.17 \pm 0.05$ & 1 & 1 & 1 & 1 & 1 & 1 & 1 & 1 & 1 & 1 & 1 & 1 & 1 & 1 \\
\hline F39 & $\begin{array}{l}\text { Kaempferol-3-O-glycoside } \\
\text {-7-sustituted }\end{array}$ & $42.56 \pm 0.02$ & 0 & 0 & 1 & 1 & 0 & 1 & 0 & 1 & 0 & 0 & 0 & 0 & 0 & 0 \\
\hline F40 & Kaempferol-3-O-glycoside & $42.57 \pm 0.05$ & 1 & 1 & 0 & 0 & 1 & 0 & 1 & 1 & 1 & 1 & 1 & 1 & 1 & 1 \\
\hline F41 & Phenolic acid & $42.63 \pm 0.00$ & 1 & 0 & 0 & 0 & 0 & 0 & 0 & 0 & 0 & 0 & 0 & 0 & 0 & 0 \\
\hline F42 & Phenolic acid & $42.90 \pm 0.01$ & 1 & 0 & 1 & 1 & 1 & 1 & 1 & 1 & 1 & 0 & 0 & 0 & 0 & 0 \\
\hline F43 & Phenolic acid & $43.19 \pm 0.01$ & 1 & 0 & 0 & 0 & 0 & 0 & 0 & 0 & 1 & 1 & 1 & 1 & 1 & 1 \\
\hline F44 & Phenolic acid & $43.92 \pm 0.02$ & 0 & 1 & 1 & 1 & 1 & 1 & 1 & 1 & 0 & 0 & 0 & 0 & 0 & 0 \\
\hline F45 & Phenolic acid & $44.17 \pm 0.00$ & 1 & 0 & 0 & 0 & 0 & 0 & 0 & 0 & 1 & 1 & 1 & 1 & 1 & 1 \\
\hline F46 & Phenolic acid & $53.30 \pm 0.02$ & 1 & 1 & 1 & 1 & 1 & 1 & 1 & 1 & 1 & 1 & 1 & 1 & 1 & 1 \\
\hline & Total & 22 & 19 & 14 & 15 & 16 & 16 & 15 & 16 & 16 & 17 & 16 & 16 & 16 & 15 & 15 \\
\hline
\end{tabular}

X: Mean; SD: Standard deviation; 1: Found; 0: Not found

Table 4: Major phenols found in the pollen of Mammillaria heyderi sensu lato

\begin{tabular}{|c|c|c|c|c|c|c|c|c|c|c|c|c|c|c|}
\hline \multirow{2}{*}{$\begin{array}{l}\text { No. of } \\
\text { compound }\end{array}$} & \multirow[b]{2}{*}{ Compound } & \multirow{2}{*}{$\begin{array}{l}\text { Retention } \\
\text { time }(\mathrm{min}) \\
(\mathrm{X} \pm \mathrm{SD})\end{array}$} & \multicolumn{12}{|c|}{$\begin{array}{l}\text { Samples } \\
\text { Mammillaria heyderi sensu lato }\end{array}$} \\
\hline & & & 567 & 576 & 577 & 578 & 579 & 580 & 581 & 582 & 583 & 584 & 585 & 586 \\
\hline$\overline{\text { F6 }}$ & $\begin{array}{l}\text { Kaempferol-3-O-glycoside } \\
\text {-7-substituted }\end{array}$ & $32.56 \pm 0.020$ & 1 & 1 & 1 & 1 & 1 & 1 & 1 & 1 & 1 & 1 & 1 & 1 \\
\hline F7 & Phenolic acid & $32.68 \pm 0.050$ & 1 & 1 & 1 & 1 & 1 & 1 & 1 & 1 & 1 & 1 & 1 & 1 \\
\hline F10 & Phenolic acid & $34.18 \pm 0.010$ & 1 & 1 & 1 & 1 & 1 & 1 & 1 & 1 & 1 & 1 & 1 & 1 \\
\hline F12 & Herbacetin-3-O-glycoside & $36.32 \pm 0.030$ & 1 & 1 & 1 & 1 & 1 & 1 & 1 & 1 & 1 & 1 & 1 & 1 \\
\hline F13 & Herbacetin-3-O-glycoside & $36.35 \pm 0.030$ & 1 & 1 & 1 & 1 & 1 & 1 & 1 & 1 & 1 & 1 & 1 & 1 \\
\hline F16 & Unidentified flavonol & $36.80 \pm 0.000$ & 0 & 0 & 1 & 1 & 1 & 0 & 0 & 1 & 0 & 0 & 0 & 1 \\
\hline F18 & Herbacetin-3-O-glycoside & $37.54 \pm 0.020$ & 1 & 1 & 1 & 1 & 1 & 1 & 1 & 1 & 1 & 1 & 1 & 1 \\
\hline F19 & Herbacetin-3-O-glycoside & $37.76 \pm 0.000$ & 1 & 0 & 0 & 1 & 1 & 0 & 0 & 0 & 1 & 0 & 0 & 0 \\
\hline F22 & Unidentified flavonoid & $37.82 \pm 0.010$ & 1 & 1 & 1 & 1 & 1 & 0 & 1 & 1 & 1 & 0 & 0 & 0 \\
\hline F25 & Kaempferol-3-O-glycoside & $38.24 \pm 0.020$ & 1 & 1 & 1 & 1 & 1 & 1 & 1 & 1 & 1 & 1 & 1 & 1 \\
\hline F30 & Kaempferol-3-O-glycoside & $39.12 \pm 0.050$ & 1 & 1 & 1 & 1 & 1 & 1 & 1 & 1 & 1 & 1 & 1 & 1 \\
\hline F33 & Kaempferol-3-O-glycoside & $39.88 \pm 0.026$ & 1 & 1 & 1 & 1 & 1 & 1 & 1 & 1 & 1 & 1 & 1 & 1 \\
\hline F35 & Quercetin-3-O-glycoside & $40.1 \pm 0.0400$ & 1 & 1 & 1 & 0 & 1 & 1 & 1 & 1 & 1 & 1 & 0 & 1 \\
\hline F36 & Quercetin-3-O-glycoside & $40.56 \pm 0.020$ & 1 & 1 & 1 & 1 & 1 & 1 & 1 & 1 & 1 & 1 & 1 & 1 \\
\hline F37 & Kaempferol-3-O-glycoside & $41.17 \pm 0.050$ & 1 & 1 & 1 & 1 & 1 & 1 & 1 & 1 & 1 & 1 & 1 & 1 \\
\hline F38 & Quercetin-3-O-glycoside & $41.55 \pm 0.020$ & 1 & 1 & 0 & 0 & 1 & 1 & 0 & 1 & 1 & 1 & 0 & 1 \\
\hline \multirow[t]{2}{*}{ F40 } & Kaempferol-3-O-glycoside & $42.57 \pm 0.050$ & 1 & 1 & 1 & 1 & 1 & 1 & 1 & 1 & 1 & 1 & 1 & 1 \\
\hline & Total & 17 & 16 & 15 & 15 & 15 & 17 & 14 & 14 & 16 & 16 & 14 & 12 & 15 \\
\hline
\end{tabular}

Species of Cactaceae investigated for their composition of phenolics in the tepals, as a part of perianth, are Astrophytum ornatum Web., Notocactus apricus A.
Berg., Echinopsis huotii Lab., Aylostera pseudodeminuta Backbg. and Neochilenia napina Backberg.; in them no dihydroflavonol was detected ${ }^{[22]}$. 
Am. J. Agri. \& Biol. Sci., 3 (3): 534-543, 2008

Table 5: Major phenols found in the pollen of Echinocereus pectinatus

\begin{tabular}{|c|c|c|c|c|c|c|c|c|c|c|}
\hline \multirow{2}{*}{$\begin{array}{l}\text { No. of } \\
\text { compound }\end{array}$} & \multirow[b]{2}{*}{ Compound } & \multirow{2}{*}{$\begin{array}{l}\text { Retention } \\
\text { time (min) } \\
(\mathrm{X} \pm \mathrm{SD})\end{array}$} & \multicolumn{8}{|c|}{$\begin{array}{l}\text { Samples } \\
\text { Echinocereus pectinatus }\end{array}$} \\
\hline & & & 630 & 631 & 632 & 634 & 635 & 636 & 637 & 658 \\
\hline F1 & Unidentified phenol & $25.73 \pm 0.010$ & 1 & 0 & 0 & 0 & 1 & 1 & 1 & 1 \\
\hline $\mathrm{F} 2$ & Unidentified phenol & $29.93 \pm 0.030$ & 1 & 0 & 0 & 0 & 1 & 1 & 1 & 1 \\
\hline F3 & Unidentified phenol & $30.65 \pm 0.020$ & 1 & 0 & 0 & 1 & 1 & 1 & 0 & 1 \\
\hline F4 & Kaempferol-3-O-glycoside & $32.24 \pm 0.020$ & 1 & 0 & 0 & 1 & 1 & 1 & 1 & 1 \\
\hline F8 & Kaempferol-3-O-glycoside & $33.91 \pm 0.000$ & 1 & 0 & 0 & 1 & 1 & 1 & 1 & 0 \\
\hline F9 & Herbacetin-3-O-glycoside & $34.16 \pm 0.020$ & 1 & 1 & 1 & 1 & 1 & 1 & 1 & 1 \\
\hline F11 & Phenolic acid & $35.73 \pm 0.090$ & 1 & 1 & 1 & 1 & 1 & 1 & 1 & 1 \\
\hline F13 & Herbacetin-3- $O$-glycoside & $36.35 \pm 0.030$ & 1 & 1 & 1 & 1 & 1 & 1 & 1 & 1 \\
\hline F18 & Herbacetin-3-O-glycoside & $37.54 \pm 0.020$ & 1 & 1 & 1 & 1 & 1 & 1 & 1 & 1 \\
\hline F20 & Quercetin-3-O-glycoside & $37.77 \pm 0.030$ & 1 & 1 & 1 & 1 & 1 & 1 & 1 & 1 \\
\hline $\mathrm{F} 21$ & Kaempferol-3-O-glycoside & $37.80 \pm 0.010$ & 1 & 1 & 1 & 1 & 1 & 1 & 1 & 1 \\
\hline F24 & $\begin{array}{l}\text { Kaempferol-3-O-glycoside } \\
\text { (probably substituted in ring A) }\end{array}$ & $38.20 \pm 0.040$ & 1 & 1 & 1 & 1 & 1 & 1 & 1 & 1 \\
\hline $\mathrm{F} 27$ & Quercetin-3-O-glycoside & $38.89 \pm 0.040$ & 1 & 1 & 1 & 1 & 1 & 1 & 1 & 1 \\
\hline F30 & Kaempferol-3-O-glycoside & $39.12 \pm 0.050$ & 1 & 1 & 1 & 1 & 1 & 1 & 1 & 1 \\
\hline F33 & Kaempferol-3-O-glycoside & $39.88 \pm 0.026$ & 1 & 1 & 1 & 1 & 1 & 1 & 1 & 1 \\
\hline F37 & Kaempferol-3-O-glycoside & $41.17 \pm 0.050$ & 1 & 1 & 1 & 1 & 1 & 1 & 1 & 1 \\
\hline \multirow[t]{2}{*}{ F40 } & Kaempferol-3- $O$-glycoside & $42.57 \pm 0.050$ & 1 & 1 & 1 & 1 & 1 & 1 & 1 & 1 \\
\hline & Total & 17 & 17 & 12 & 12 & 15 & 17 & 17 & 16 & 16 \\
\hline
\end{tabular}

X: Mean; SD: Standard deviation; 1: Found; 0: Not found

Table 6: Major phenols found in the pollen of Echinocereus enneacanthus

\begin{tabular}{|c|c|c|c|c|c|c|c|c|c|c|c|c|c|}
\hline \multirow{2}{*}{$\begin{array}{l}\text { No. of } \\
\text { Compound }\end{array}$} & \multirow{2}{*}{ Compound } & \multirow{2}{*}{$\begin{array}{l}\text { Retention } \\
\text { time }(\min ) \\
(\bar{X} \pm S D)\end{array}$} & \multicolumn{11}{|c|}{$\begin{array}{l}\text { Samples } \\
\text { Echinocereus enneacanthus }\end{array}$} \\
\hline & & & 609 & 610 & 611 & 612 & 638 & 639 & 641 & 643 & 644 & 645 & 646 \\
\hline F9 & Herbacetin-3-O-glycoside & $34.16 \pm 0.02$ & 1 & 1 & 1 & 1 & 1 & 1 & 1 & 1 & 1 & 1 & 1 \\
\hline F15 & Kaempferol-3-O-glycoside & $36.56 \pm 0.04$ & 0 & 0 & 0 & 0 & 0 & 0 & 0 & 0 & 0 & 1 & 0 \\
\hline F18 & Herbacetin-3-O-glycoside & $37.54 \pm 0.02$ & 0 & 0 & 0 & 0 & 0 & 0 & 0 & 1 & 0 & 1 & 0 \\
\hline F23 & Quercetin-3-O-glycoside & $37.88 \pm 0.04$ & 1 & 1 & 1 & 1 & 1 & 1 & 1 & 1 & 1 & 1 & 1 \\
\hline $\mathrm{F} 25$ & Kaempferol-3-O-glycoside & $38.24 \pm 0.02$ & 1 & 1 & 1 & 1 & 1 & 1 & 1 & 1 & 1 & 1 & 1 \\
\hline F27 & Quercetin-3-O-glycoside & $38.89 \pm 0.04$ & 1 & 1 & 1 & 1 & 1 & 1 & 1 & 1 & 1 & 1 & 1 \\
\hline F33 & Kaempferol-3-O-glycoside & $39.88 \pm 0.026$ & 1 & 1 & 1 & 1 & 1 & 1 & 1 & 1 & 1 & 1 & 1 \\
\hline F37 & Kaempferol-3-O-glycoside & $41.17 \pm 0.05$ & 1 & 1 & 1 & 1 & 1 & 1 & 1 & 1 & 1 & 1 & 1 \\
\hline \multirow[t]{2}{*}{ F40 } & Kaempnferol-3-O-glycoside & $42.57 \pm 0.05$ & 1 & 1 & 1 & 1 & 1 & 1 & 1 & 1 & 1 & 1 & 1 \\
\hline & Total & 9 & 7 & 7 & 7 & 7 & 7 & 7 & 7 & 8 & 7 & 9 & 7 \\
\hline
\end{tabular}

X: Mean; SD: Standard deviation; 1: Found; 0: Not found

Among the four species analyzed, Stenocactus multicostatus subs. zacatecasensis had the pollen phenolic profile most complex (Table 3), with 22 structures: 1 unidentified flavonoid, 8 phenolic acid derivatives and 13 flavonol glycosides, from which 2 are gycosilherbacetin derivatives, 2 are glycosilquercetin derivatives and 9 are glycosilkaempferol derivatives. The most simple pollen phenolic profile was that of Echinocereus enneacanthus (Table 6), with 9 structures, 2 glycosilherbacetin derivatives, 2 glycosilquercetin derivatives and 5 glicosilkaempferol derivatives.

Taxonomic analysis: Twelve phenolics (F5, F13, F14, F17, F18, F23, F26, F27, F31, F34, F37 and F46) among a total of 22 were always present in the pollen of every individual identified as Stenocactus multicostatus subsp. zacatecasensis; 7 (F9, F23, F25, F27, F33, F37, F40) among a total of 9 were present in every individual of Echinocereus enneacanthus; 12 (F9, F11, F13, F18, F20, F21, F24, F27, F30, F33, F37, F40) among a total of 17 were always present in Echinocereus pectinatus; while 12 phenolics (F6, F7, F10, F12, F13, F18, F25, F30, F33, F36, F37 and F40) among a total of 17 were present in the pollen of every analyzed individual of Mammillaria heyderi sensu lato. These figures suggest that the pollen of every these four species of Cactaceae synthesizes more than the fifty per cent of its phenolics in a very conservative way.

With regard to the richness of the various classes of the flavonoid/phenolic acid profiles as expressed by the Shannon index for each taxon, our results suggest that 
Mammillaria heyderi sensu lato and Stenocactus multicostatus subsp. zacatecasensis had the relative highest variability $(\mathrm{H}=2.254$ and $\mathrm{H}=2.107$, respectively), that Echinocereus pectinatus has an intermediate variability $(\mathrm{H}=1.494)$ and that E. enneacanthus was the taxa most homogeneous $(\mathrm{H}=0.600)$. These figures represent the natural intrapopulation variability in the expression of pollen phenolic profiles in each taxon studied. This kind of variability has been reported for the foliar phenolic profiles of Aspalathus linearis ${ }^{[23]}$ and for five species of Pinus $^{[11,12]}$. In all those taxa, the phenolic profiles were significant markers for delimiting species.
Despite the observed variability, each analyzed taxon displays a unique flavonoid/phenolic acid profile (Table 7). Fifteen compounds (F5, F14, F17, F26, F29, F31, F32, F34, F39, F41, F42, F43, F44, F45 and F46) were characteristic of Stenocactus multicostatus subsp. zacatecasensis, one (F15) were found only in individuals of Echinocereus enneacanthus, 8 (F1, F2, F3, F4, F8, F11, F20 and F21) were exclusive of Echinocereus pectinatus and 10 (F6, F7, F10, F12, F16, F19, F22, F35, F36 and F38) were present solely in individuals of Mammillaria heyderi sensu lato. of a total of 46 compounds, three (F18, F37 and F40) were common to all analyzed taxa.

Table 7: Taxonomic distribution of pollen flavonoid/phenolic acid compounds in Stenocactus multicostatus subsp zacatecasensis, Mammillaria heyderi sensu lato, Echinocereus pectinatus and E. enneacanthus

\begin{tabular}{|c|c|c|c|c|c|}
\hline $\begin{array}{l}\text { No.of } \\
\text { compound }\end{array}$ & Chemical identification & $\begin{array}{l}\text { Stenocactus multicostatus } \\
\text { subsp. zacatecasensis }\end{array}$ & $\begin{array}{l}\text { Mammillaria } \\
\text { heyderi sensu lato }\end{array}$ & $\begin{array}{l}\text { Echinocereus } \\
\text { pectinatus }\end{array}$ & $\begin{array}{l}\text { Echinocereus } \\
\text { enneacanthus }\end{array}$ \\
\hline F1 & Unidentified phenol & - & - & $+/-$ & - \\
\hline $\mathrm{F} 2$ & Unidentified phenol & - & - & $+/-$ & - \\
\hline F3 & Unidentified phenol & - & - & $+/-$ & - \\
\hline $\mathrm{F} 4$ & Kaempferol-3-O-glycoside & - & - & $+/-$ & - \\
\hline F5 & Kaempferol-3-O-glycoside & + & - & - & - \\
\hline F6 & Kaempferol-3-O-glycoside-7-substituted & - & + & - & - \\
\hline F7 & Phenolic acid & - & $+/-$ & - & - \\
\hline F8 & Kaempferol-3-O-glycoside & - & - & $+/-$ & - \\
\hline F9 & Herbacetin-3-O-glycoside & - & - & $+/-$ & $+/-$ \\
\hline F10 & Phenolic acid & - & + & - & - \\
\hline F11 & Phenolic acid & - & - & + & - \\
\hline $\mathrm{F} 12$ & Herbacetin-3-O-glycoside & - & $+/-$ & - & - \\
\hline F13 & Herbacetin-3-O-glycoside & + & + & $+/-$ & - \\
\hline F14 & Phenolic acid & + & - & - & - \\
\hline F15 & Kaempferol-3-O-glycoside & - & - & - & $+/-$ \\
\hline F16 & Unidentified flavonol & - & $+/-$ & - & - \\
\hline F17 & Quercetin-3-O-glycoside & + & - & - & - \\
\hline F18 & Herbcetin-3-O-glycoside & + & + & + & $+/-$ \\
\hline F19 & Herbacetin-3-O-glycoside & - & $+/-$ & - & - \\
\hline $\mathrm{F} 20$ & Quercetin-3-O-glycoside & - & - & + & - \\
\hline $\mathrm{F} 21$ & Kaempferol-3-O-glycoside & - & - & + & - \\
\hline $\mathrm{F} 22$ & Unidentified flavonoid & - & $+/-$ & - & - \\
\hline $\mathrm{F} 23$ & Quercetin-3-O-glycoside & + & - & - & + \\
\hline $\mathrm{F} 24$ & $\begin{array}{l}\text { Kaempferol-3-O-glycoside } \\
\text { (probably substituted in ring A) }\end{array}$ & $+/-$ & - & + & - \\
\hline $\mathrm{F} 25$ & Kaempferol-3-O-glycoside & - & + & - & $+/-$ \\
\hline F26 & Kaempferol-3-O-glycoside & + & - & - & - \\
\hline $\mathrm{F} 27$ & Quercetin-3-O-glycoside & + & - & + & + \\
\hline F28 & Unidentified flavonol & - & - & - & - \\
\hline F29 & Phenolic acid & $+/-$ & - & - & - \\
\hline $\mathrm{F} 30$ & Kaempferol-3-O-glycoside & - & + & $+/-$ & - \\
\hline F31 & Kaempferol-3-O-glycoside & + & - & - & - \\
\hline F32 & Unidentified flavonol & $+/-$ & - & - & - \\
\hline $\mathrm{F} 33$ & Kaempferol-3-O-glycoside & - & + & + & + \\
\hline $\mathrm{F} 34$ & Kaempferol-3-O-glycoside & + & - & - & - \\
\hline F35 & Quercetin-3-O-glycoside & - & $+/-$ & - & - \\
\hline F36 & Quercetin-3-O-glycoside & - & + & - & - \\
\hline F37 & Kaempferol-3-O-glycoside & + & + & + & + \\
\hline F38 & Quercetin-3-O-glycoside & - & $+/-$ & - & - \\
\hline F39 & Kaempferol-3-O-glycoside-7-substituted & $+/-$ & - & - & - \\
\hline F40 & Kaempferol-3-O-glycoside & $+/-$ & + & + & + \\
\hline $\mathrm{F} 41$ & Phenolic acid & $+/-$ & - & - & - \\
\hline $\mathrm{F} 42$ & Phenolic acid & $+/-$ & - & - & - \\
\hline $\mathrm{F} 43$ & Phenolic acid & $+/-$ & - & - & - \\
\hline $\mathrm{F} 44$ & Phenolic acid & $+/-$ & - & - & - \\
\hline F45 & Phenolic acid & $+/-$ & - & - & - \\
\hline F46 & Phenolic acid & + & - & - & - \\
\hline
\end{tabular}




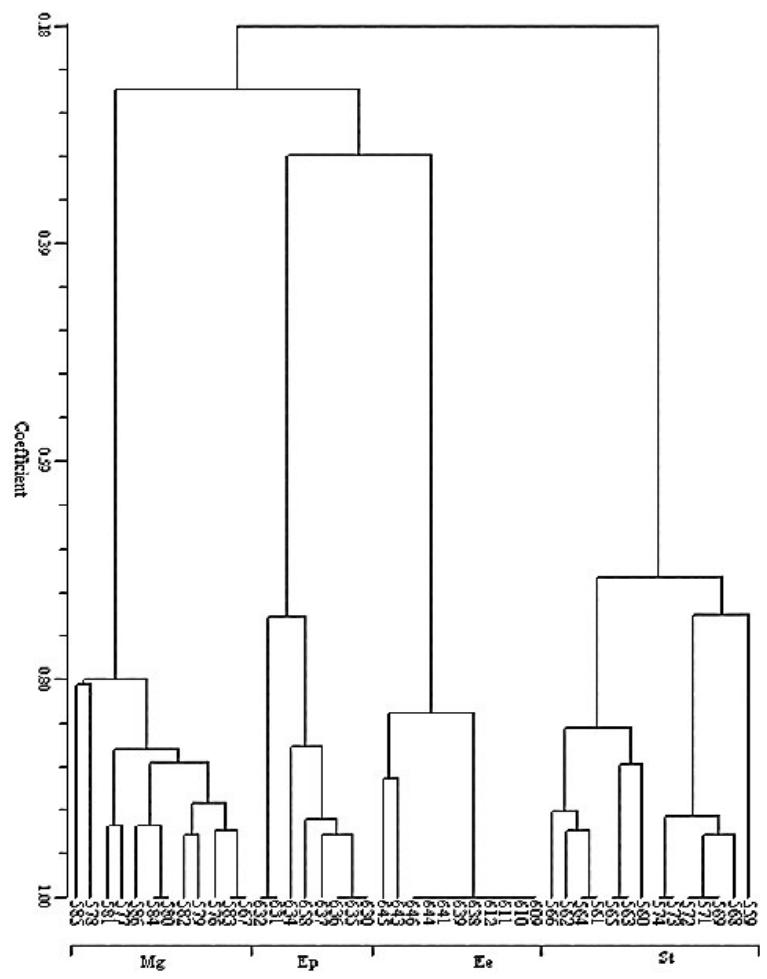

Fig. 1: Results of clustering analysis comparing pollen phenolic profiles for 45 samples of Cactaceae (Mg: Mammillaria heyderi sensu lato, Ep: Echinocereus pectinatus, Ee: Echinocereus enneacanthus, St: Stenocactus multicostatus subsp. zacatecasensis)

Variation among groups of individuals associated with environmental variables such as elevation, latitude or particular site was not observed. Pollen flavonoid/phenolic acid profiles of individuals of Echinocereus enneacanthus, which came from two different populations, separated one each from the other by around $200 \mathrm{Km}$ and by orographic barriers as the Sierra El Rosario, with elevations reaching $2240 \mathrm{~m}$, formed a group without subdivisions separating these two populations (Fig. 1). This supports suggestions by Kaundun et al. ${ }^{[24]}$, Almaraz-Abarca et al. ${ }^{[12]}$ and others that flavonoid composition is little affected by environmental fluctuations.

Mammillaria heyderi sensu lato is a complicated taxonomic group due to high polymorphism and hybridizing capacity ${ }^{[5]}$. This taxon and Echinocereus pectinatus both are distributed through a very broad zone, practically in a continuous way in State of Durango, Mexico. They are found either in arid zones or in template Pinus-Quercus forest. Their distributions contrast with that of Stenocactus multicostatus subsp. zacatecasensis, which is found in scarce and disjunctive populations ${ }^{[25]}$. These three species can be found as sympatric populations in zones of Quercus forest with few grasses and rocky substratum, like it is the growing habitats of Stenocactus multicostatus subsp. zacatecasensis. Mammillaria heyderi sensu lato and Echinocereus pectinatus both with a broad distribution, showed a relative high flavonoid/phenolic acid variability in pollen $(\mathrm{H}=2.254$ and $\mathrm{H}=1.494$, respectively). These results were expected, since according to Sosa et al. ${ }^{[26]}$ the increment in the morphological and genetic variability is associated to the increment in the distribution area. However, the high variability in the phenolic composition of pollen of Stenocactus multicostatus subsp. zacatecasensis $(\mathrm{H}=2.107)$, which is similar to that of Mammillaria heyderi sensu lato, can not be associated to a broad distribution, but to a high intrapopulation genetic variability. The high chemical variability found in the pollen phenol composition of Stenocactus multicostatus subs. zacatecasensis is according to the Bravo-Hollis and Sánchez-Mejorada ${ }^{[5]}$, statement about this taxon is in a present speciation process; in this taxon both chemical and morphological variability may be maintained by a heterogeneous environmental pressure of selection.

The fenetic analysis of pollen phenol profiles groups together Mammillaria heyderi sensu lato (Tribe Cacteae) and the two species of Echinocereus (Tribe Echinocereae) and place Stenocactus multicostatus subsp. zacatecasensis in a separated group, (Fig. 1). However, on a morphological basis, Stenocactus multicostatus subsp. zacatecasensis shares along to Mammillaria heyderi sensu lato the same tribe Cactea $^{[5]}$. Nevertheless, in spite of the suggestion given by these results it is necessary to investigate the distribution of pollen flavonoid/phenolic acid profiles and the correlations between chemical and morphological features, among a higher number of populations, species and genera of Cactaceae before considering a closer taxonomic relationship between Mammillaria and Echinocereus than between Mammillaria and Stenocactus.

Pollen phenolic profiles confirmed taxonomic separation of the four species made on morphological basis. The fenetic analysis (Fig. 1) clearly distinguishes four groups that correspond to the four morphologically-based taxa, Stenocactus multicostatus subsp. zacatecasensis, Echinocereus enneacanthus, Echinocereus pectinatus and Mammillaria heyderi sensu lato. This indicates that each of four species can be distinguished from the others by a unique flavonoid/phenolic acid profile. Pollen phenolic profiles 
are species-specific and each species express a variable number of unique flavonoids or phenolic acid.

In spite of the intrapopulation variability, the pollen flavonoid/phenolic acid profiles of Stenocactus multicostatus subsp. zacatecasensis, Echinocereus enneacanthus, Echinocereus pectinatus and Mammillaria heyderi sensu lato are so stable for each species that type profiles by species can be recognized. These profiles can be considered as valuable chemical markers at the specific level. The refereed profiles, rich in quercetin, kaempferol and herbacetin glycosil derivatives, are among the most complex reported. Although more population studies on the phenolic composition of pollen of Cactaceae are needed, over all in species with a broad distribution, these results suggest that flavonoid/phenolic acid profiles of pollen could be specific taxonomic markers in this family of plants.

\section{ACKNOWLEDGMENTS}

Authors thank COFAA-IPN for stimuli to research.

\section{REFERENCES}

1. Arias, M.S., 1993. Cactáceas: conservación y diversidad en México. Rev. Soc. Mex. Hist. Nat., 44: 109-115.

2. Barthlott, W. and D.R. Hunt, 1993. Cactaceae. In: The Families and Genera of Vascular Plants, Kubitzki, K., J.G. Rohwer and V. Bittrich (Eds.). Springer Verlag, Berlin, pp: 161-197.

3. Arias, M.S., 2001. Sistemática y conservación de la familia Cactaceae en México. Memorias del XV Congreso Mexicano de Botánica, México.

4. Arias, M.S., 1997. Distribución General. In: Suculentas Mexicanas. Cactáceas, Valles, S.C. and P.L. Rodríguez (Eds.). UNAM, SEMARNAP, México, pp: 17-25.

5. Bravo-Hollis, H. and R.H. Sánchez-Mejorada, 1991. Las Cactáceas de México. Volumen No. 2. UNAM, México, pp: 404.

6. Meyrán, G.J., 1979. Discusión sobre Echinofossulocactus. Cact. Suc. Mex., 24 (3): 90-94.

7. Guzmán, C.L.U., 1997. Grupos Taxonómicos. In: Suculentas Mexicanas. Cactaceas, Valles, S.C. and P.L. Rodríguez (Eds.). UNAM-SEMARNAP, México, pp: 37-41.

8. Abdala, L.R. and P. Seeligmann, 1995. Flavonoids in Tagetes zipaquirensis and their chemosystematic significance. Biochem. Syst. Ecol., 23 (7-8): 871-872.
9. Del Pero, M.M.A., J.P. Pelotto and N. Basualdo, 1997. Distribution of flavonoid aglycones in Ilex species (Aquifoliaceae). Biochem. Syst. Ecol., 25 (7): 619-622.

10. Fiasson, J.L., K. Gluchoff-Fiasson and G. Dahlgren, 1997. Flavonoid patterns in European Ranunculus L. subgenus Batrachium (Ranunculaceae). Biochem. Syst. Ecol., 25 (4): 327-333.

11. Almaraz-Abarca, N., 2000. Estudio quimiotaxonómico de Pinus sección Leiophyllae (Pinaceae). Tesis Doctoral. Escuela Nacional de Ciencias Biológicas, Instituto Politécnico Nacional, México, pp: 157.

12. Almaraz-Abarca, N., M.S. González-Elizondo, J.A. Tena-Flores, J.A. Ávila-Reyes, J. HerreraCorral and N. Naranjo-Jiménez, 2006. Foliar flavonoids distinguish Pinus leiophylla and Pinus chihuahuana (Coniferales: Pinaceae). Proc. Biol. Soc. Wash., 119 (3): 426-436.

13. Wiermann, R. and K. Vieth, 1983. Outer pollen wall, an important accumulation site for flavonoids. Protoplasma, 118 (3): 230-233.

14. Markham, K. and M.G. Campos, 1996. 7- and 8-Omethylherbacetin-3- $O$-sophoroside from bee pollens and some struture/activity observations. Phytochem, 43 (4): 762-767.

15. Almaraz-Abarca, N., M.G. Campos, J.A. ÁvilaReyes, N. Naranjo-Jiménez, J. Herrera-Corral and L.S. González-Valdez, 2004a. Variability of antioxidant activity among honeybee-collected pollen of different botanical origin. Interciencia, 29 (10): 574-578.

16. Campos, M.G. and K.R. Markham, 2007. Structure information from HPLC and on-line measured absortion spectra-flavone, flavonols and phenolic acids. Coimbra University Press, Portugal, pp: 118.

17. Rohlf, F.J., 1993. NTSyS-pc Version 1.8. Numerical taxonomy and multivariate analysis system.

18. Ylstra, B., T. Alisher, M.R.M. Benito, E. Stöger, A.J. van Tunen, O. Vicente, J.N.M. Mol and E. Heberle-Bors, 1992. Flavonols stimulate development, germination and tube growth of tobacco pollen. Plant Physiol., 100 (2): 902-907.

19. Mo, Y., C. Ángel and L.P. Taylor, 1992. Biochemical complementation of chalcone synthase mutants defines a role for flavonols in functional pollen. Proc. Nat. Acad. Sci., 89 (15): 7213-7217.

20. Burbulis, I.E., M. Iacobucci and B.W. Shirley, 1996. A null mutation in the first enzyme of flavonoid biosíntesis does not affect male fertility in Arabidopsis. Plant Cell, 8 (6): 1013-1025. 
21. Miller, J.M. and B.A. Bohmt, 1982. Flavonol and dihydroflavonol glycosides of Echinocereus triglochidiatus var. gurneyi. Phytochem., 21 (4): 951-952.

22. Iwashina, T., S. Otan and K. Hayashi, 1986. Determination of minor flavonol-glycosides and sugar-free flavonols in the tepals of several species of Cereoideae (Cactaceae). J. Plant Res., 99 (1): 53-62.

23. Van Heerden, F.R., V.E. van Wyk, A.M. Viljoen and P.A. Steenkamp, 2003. Phenolic variation in wild populations of Aspalathus linearis (rooibos tea). Biochem. Syst. Ecol., 31 (8): 885-895.

24. Kaundun, Sh.Sh., Ph. Lebreton and B. Fady, 1998. Geographical variability of Pinus halepensis Mill as revealed by foliar flavonoids. Biochem. Syst. Ecol., 26 (1): 83-96.
25. Almaraz-Abarca, N., A. Delgado-Alvarado, J.A. Ávila-Reyes, N. Naranjo-Jiménez and J. Herrera-Corral, 2004b. Las cactáceas del Estado de Durango. Biotecipn, 2 (4): 19-20.

26. Sosa, A.P., F.J. Batista, M.A. González-Pérez and N. Bouza, 2002. La Conservación Genética de Las Especies Vegetales Amenazadas. Técnicas de Diagnóstico Del Estado de Conservación. En: Biología de la Conservación de Especies Amenazadas, Bañares, A. (Ed.). Organismo Autónomo de Parques Nacionales, Ministerio de Medio Ambiente, España, pp: 2-27. 